\title{
Depressão: Um Problema por Resolver?
}

\section{Depression: An Unsolved Problem?}

Sara Correia*, Miguel Santos*, Dilermando Sobral*

\section{Resumo}

Introdução: A depressão, síndrome multifactorial, apresenta prevalência, morbilidade e custos crescentes. Os estudos epidemiológicos sobre prevalência de perturbações mentais são escassos, tornando necessário estudos adicionais.

Objectivos: Avaliar a incidência e prevalência da depressão nos utentes da Unidade de Saúde Familiar de Ramalde (USFR), a relação entre depressão e factores demográficos e o seguimento e terapêutica farmacológica.

Métodos: Estudo observacional, transversal, retrospectivo. População: utentes com 18 ou mais anos inscritos na USFR. Amostra: utentes com diagnóstico de Perturbação Depressiva na lista de problemas activos do processo clínico. Variáveis: género, idade, anos de diagnóstico, data da última consulta, data e local da última prescrição, terapêutica farmacológica, grupo terapêutico. Instrumentos de recolha de dados: MIM@UF ${ }^{\circledR}$, SClínico $^{\circledR}$ e PEM $^{\circledR}$.

Resultados: A amostra foi de 1475 utentes (78\% mulheres). A maioria (60\%) tinha entre 40 e 69 anos. A taxa de incidência, no ano de 2014, foi de $2 \%$ e a prevalência, do total de utentes inscritos na USFR com mais de 18 anos $(n=12067)$, de $12 \%$, superior à do Agrupamento de Centros de Saúde Porto Ocidental, região norte e a nível nacional. A prevalência ajustada por género foi de $16,3 \%$ nas mulheres e de $6 \%$ nos homens e por escalões etários de 6,3\% entre os 18 e 39 anos e de $15 \%$ na faixa superior a 65 anos. A maioria teve consulta no último ano (88\%) e encontrava-se medicada com antidepressivos (82\%). A última prescrição ocorreu há mais de 6 meses em mais de metade dos casos. Os fármacos mais prescritos foram os inibidores selectivos de

\begin{abstract}
Introduction: Depression, a multifactorial syndrome, presents rising prevalence, morbidity and costs. Epidemiological studies about mental disorders prevalence are scarce, particularly in Portugal, precluding a solid assessment of this problem.
\end{abstract}

Objectives: To assess the incidence and prevalence of depression among Family Health Unit of Ramalde (FHUR) users, the relationship between depression and demographic factors, and the follow-up and drug therapy adopted by Family Physicians (FP).

Methods: Observational, cross-sectional, retrospective study. Population: users with 18 or more years old enrolled in FHUR. Sample: users diagnosed with Depressive Disorder registered in active problems' list on medical records. Variables: gender, age, year of diagnosis, date of last medical visit, date and place of last prescription, drug therapy, therapeutic group. Data collection instruments: MIM@UF ${ }^{\circledR}$, SClínico $^{\circledR}$ and PEM ${ }^{\circledR}$.

Results: Sample was composed by 1475 users (78\% women). The majority (60\%) were between 40 and 69 years old. Incidence, for the year 2014, was $2 \%$, and prevalence $12 \%$ of the patients 18 or more years old, higher than the known prevalence of Health Centers Grouping of Porto Ocidental, northern region and all country. Gender adjusted prevalence was $16.3 \%$ in women and $6 \%$ in men, and age adjusted prevalence was $6,3 \%$ in persons between 18 and 39 years old and $15 \%$, in people 65 years and older. Most subjects visited their FP in the past year (88\%) and were treated with antidepressant drugs (82\%). Time elapsed since last prescription was higher than 6 months in more than half of the cases. Most commonly prescribed drugs were selective 
recaptação da serotonina (SSRI) e 77\% das prescrições foram realizadas na USFR.

Discussão: A prevalência de depressão nesta população é alta, podendo dever-se a sobrediagnóstico. A diferença entre géneros é corroborada por outros estudos. Foi possível estabelecer populações de risco segundo a idade e género. Verificou-se que 12\% dos indivíduos diagnosticados com depressão não tinha tido consulta nos 12 meses anteriores. A maioria das prescrições correspondeu a SSRI, e foi efectuada nos Cuidados de Saúde Primários.

Palavras-chave: antidepressivos, cuidados de saúde primários, depressão, patologia depressiva.

\section{Introdução}

A depressão é uma síndrome clínica comum, de causa multifactorial, com elevada prevalência e responsável por morbilidade importante e custos sociais crescentes. Estima-se que uma em cada cinco pessoas desenvolva depressão ao longo da vida. ${ }^{1}$ Calcula-se que em 2020 os episódios de depressão major constituirão a segunda causa de anos de vida com saúde perdidos. ${ }^{2}$

Na maioria dos países, os Cuidados de Saúde Primários (CSP) são a porta de entrada para o acesso aos cuidados de saúde. Cerca de $50 \%$ das pessoas que sofrem de depressão acedem aos CSP, mas apenas numa pequena parte é feito o diagnóstico correcto e aplicada a terapêutica preconizada apesar dos tratamentos disponíveis serem efectivos e de fácil aplicabilidade. ${ }^{2}$

Em Portugal, as perturbações psiquiátricas afectam mais de um quinto da população. Deste valor global destacam-se as perturbações da ansiedade (16,5\%) e as perturbações depressivas (7,9\%). ${ }^{3}$ Um estudo recente a nivel dos CSP demonstrou que 7 a 12\% dos indivíduos apresentavam níveis "ligeiros" de stress, ansiedade e depressão, 11 a 17\% níveis "moderados", e 12 a $22 \%$ níveis "graves" ou "extremamente graves". 4

As mulheres têm maior risco de sofrer de depressão ${ }^{5-6}$, enquanto as faixas etárias mais elevadas apresentarão, segundo a literatura, menor probabilidade de sofrer de perturbações depressivas. $^{7}$

Os dados sobre o consumo de psicofármacos no nosso país evidenciam um padrão elevado de consumo, na população geral, nomeadamente de antidepressivos. Os estudos epidemiológicos de base populacional sobre serotonin reuptake inhibitors (SSRI) and $77 \%$ of prescriptions were made at Primary Care.

Discussion: Prevalence of depression in this population is high, which may be due to overdiagnosis. Differences between genders are corroborated by other studies. It was possible to determine age and gender related risk groups. About $12 \%$ of individuals were inadequately treated. Most prescriptions were SSRI, performed at Primary Care.

Key-words: antidepressant drugs, depression, depressive disorder, primary health care.

a prevalência de perturbações mentais são escassos, particularmente em Portugal ${ }^{7,8}$, pelo que são da maior importância estudos adicionais.

No âmbito destes dados e visando conhecer a realidade da Depressão na Unidade de Saúde Familiar de Ramalde (USFR), do Agrupamento de Centros de Saúde Porto Ocidental (ACeSPO), onde os investigadores exercem funções, foi desenvolvido este estudo, com os seguintes objectivos: avaliar a incidência e prevalência da depressão, analisar as diferenças entre géneros e os anos de diagnóstico, determinar os possiveis grupos populacionais com maior frequência de depressão (face às variáveis idade e género), avaliar o seguimento destes doentes (avaliação quantitativa - número de consultas), e qual a distribuição da terapêutica farmacológica adoptada.

\section{Métodos}

Trata-se de um estudo observacional, transversal e retrospectivo, cuja população era composta pelos 12067 utentes inscritos na USFR, com idade igual ou superior a 18 anos.

A prevalência (número total de casos) foi calculada com base no número de utentes com o diagnóstico de Perturbação Depressiva (P76) segundo a Classificação Internacional de Cuidados Primários - $2^{a}$ Edição (ICPC2) na lista de problemas activos do processo clínico electróniCo (SClínico $\left.{ }^{\circledR}\right)$, cumulativamente, à data de 31.12.2014. A taxa de incidência (número de novos casos), foi calculada para o ano de 2014, com base no número de novos diagnósticos registados na mesma codificação. 
As variáveis utilizadas neste estudo foram: género (variável dicotonómica - feminino/masculino), idade (variável contínua), anos de diagnóstico de depressão (variável contínua), data da última consulta registada na USFR, data da última prescrição, local da última prescrição (variável dicotonómica - USF/Externo), terapêutica antidepressiva (variável dicotonómica - sim/não), e grupo terapêutico utilizado (ADT - antidepressivos tricíclicos, AgoM - agonista melatoninérgico, NaSSA - noradrenérgicos e serotonérgicos específicos, NDRI - inibidores de recaptação de dopamina e noradrenalina, Outros tianeptina ou maprotilina, RIMA - inibidor reversível da MAO (monoaminooxidade), SARI - agonistas da serotonina e inibidores da sua recaptação, SNRI - inibidores da recaptação de serotonina e noradrenalina, ou SSRI - inibidores seletivos da recaptação de serotonina).

Os dados foram recolhidos pelos investigadores na USFR através da consulta do MIM@UF $^{\circledR}$ (Módulo de Informação e Monitorização das Unidades Funcionais), do SClínico ${ }^{\circledR}$ e da PEM ${ }^{\circledR}$ (Prescrição Eletrónica Médica), durante os meses de Abril e Maio de 2015, e posteriormente introduzidos e analisados numa base de dados informática, utilizando o software Statistical Package for the Social Sciences (SPSS ${ }^{\circledR}$, versão 20.0).

O SClínico ${ }^{\circledR}$ é o sistema informático desenvolvido pelos Serviços Partilhados do Ministério da Saúde (SPMS) para as instituições do Serviço Nacional de Saúde, com as funções de agenda e de processo clínico electrónico, permitindo o registo através do método de Weed. A $\mathrm{PEM}^{\circledR}$ é o módulo de prescrição electrónica igualmente desenvolvido pelos SPMS. O MIM@UF ${ }^{\circledR}$ é o módulo estatístico para exploração de dados a nível local, no contexto das unidades funcionais dos CSP.

\section{Resultados}

O total de utentes com idade igual ou superior a 18 anos com diagnóstico de Perturbação Depressiva na lista de problemas activos do processo clínico electrónico (SClínico $\left.{ }^{\circledR}\right)$, à data de 31.12.2014, era de 1475 utentes.

A prevalência de Perturbação Depressiva na USFR, calculada à data de 31.12.2014, era de $12 \%$, superior aos valores conhecidos relativos ao ACeSPO, a que a unidade pertence $(10,1 \%)$ (segundo dados do SIARS ${ }^{\circledR}$ 2014), à da zona norte do país (8,3\%) ou à que se verifica a nível nacional (7,9\%) (gráfico 1), de acordo com os valores da Direcão Geral de Saúde. ${ }^{7}$ No entanto, não é possível obter um significado estatístico sobre esta diferença, uma vez que não se conhecem os intervalos de confiança para as estimativas de prevalência apresentadas a nível nacional e regional.

Gráfico 1. Prevalência de depressão em Portugal, Região Norte, ACeSPO e USFR ${ }^{7}$

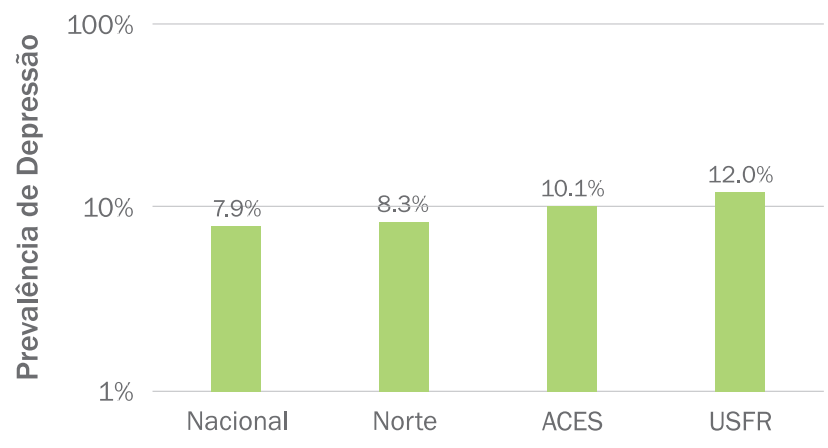

Da análise dos dados, conclui-se que a taxa de incidência em 2014 foi de 1,8\%, que corresponde aos 218 casos diagnosticados em 2014. Relativamente a este valor, pode-se afirmar que se tem mantido relativamente estável, atendendo ao número de casos diagnosticados nos 5 anos prévios à data do estudo, conforme evolução apresentada no gráfico 2.

Gráfico 2. Distribuição da amostra segundo anos de diagnóstico activo

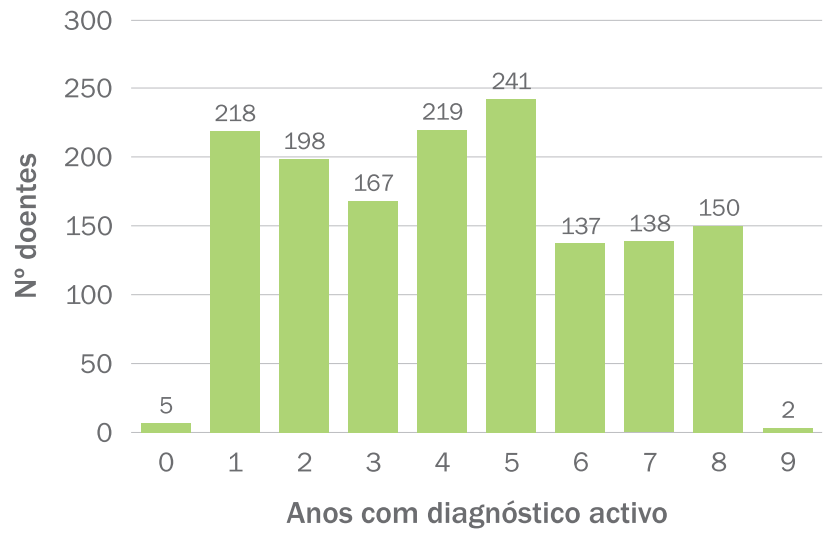

Neste estudo, 1150 utentes com codificação de Perturbação Depressiva eram do género feminino e 325 do masculino. É preciso notar que, para além de constituírem a maior fatia de utentes com este diagnóstico (78\%), as mulheres representam também a maioria (58,2\%) dos utentes com idade igual ou superior a 18 anos, motivo pelo qual foi efectuado o ajuste da prevalência por sexos. Após este ajuste, a proporção encontrada entre mulheres e homens na amostra deste estudo foi de 2,7 para 1 , com uma prevalência de depressão, na população total da USFR, de 16,3\% (1150/7034) nas mulheres e de $6 \%$ (325/5033) nos homens. 
Tal como para os géneros, também para a variável 'idade' os dados relativos à prevalência de Depressão foram ajustados (por faixa etária), por padronização directa, mediante a pirâmide etária da USFR. Assim, cerca de $60 \%$ dos utentes com diagnóstico de depressão têm idades compreendidas entre os 40 e os 69 anos (tabela 1).

Aproximadamente $70 \%$ dos indivíduos em estudo têm diagnóstico activo há menos de 5 anos $(n=1048)$. Cerca de 30\% apresentam diagnóstico activo há mais de 5 anos, alguns destes desde o início dos registos informáticos (n=457) (gráfico 2).

Tabela 1. Distribuição da amostra por género e idade

\begin{tabular}{cccc} 
Idade & Homem $n(\%)$ & Mulher $n(\%)$ & Total $n(\%)$ \\
\hline $\mathbf{2 0}$ & $2(26,0)$ & $11(74,0)$ & $13(0,9)$ \\
\hline $20-29$ & $17(25,0)$ & $51(75,0)$ & $68(4,6)$ \\
\hline $30-39$ & $43(21,9)$ & $153(78,1)$ & $196(13,3)$ \\
\hline $40-49$ & $67(22,1)$ & $236(77,9)$ & $303(20,5)$ \\
\hline $50-59$ & $64(21,3)$ & $236(78,7)$ & $300(20,3)$ \\
\hline $60-69$ & $62(22,8)$ & $210(77,2)$ & $272(18,5)$ \\
\hline $70-79$ & $41(22,4)$ & $142(77,6)$ & $183(12,4)$ \\
\hline $80-89$ & $25(21,4)$ & $92(78,6)$ & $117(7,9)$ \\
\hline$>90$ & $4(17,4)$ & $19(82,6)$ & $23(1,6)$ \\
\hline Total & $325(22,0)$ & $1150(78,0)$ & 1475
\end{tabular}

Uma grande maioria dos indivíduos diagnosticados (88\%) teve consulta presencial nos 12 meses prévios à data da recolha dos dados deste estudo; no entanto, uma percentagem não desprezivel (aproximadamente 12\%) não tinha consulta há mais de um ano.

Cerca de $82 \%$ dos utentes com diagnóstico de depressão activo estava ou já tinha sido medicado com terapêutica farmacológica antidepressiva, mas cerca de 18\% não tinham registo de qualquer prescrição com terapêutica farmacológica antidepressiva no registo clínico electrónico. A maior fatia das prescrições daqueles fármacos foi realizada a nível dos cuidados de saúde primários (USFR) - aproximadamente 77\% (tabela 2).

Tabela 2. Prescrição de terapêutica antidepressiva e local da última prescrição

\begin{tabular}{c|c|c|c}
\hline Prescrição & Homens $\mathrm{n}(\%)$ & Mulheres $\mathrm{n}(\%)$ & Total $\mathrm{n}(\%)$ \\
\hline Não & $80(29,7)$ & $179(69,1)$ & $259(17,6)$ \\
\hline Sim & $245(20,1)$ & $971(79,9)$ & $1216(82,4)$ \\
\hline & Local da última prescrição & \\
\hline USF & - & - & $939(77,2)$ \\
\hline Externo & - & - & $277(22,8)$ \\
\hline Total & & & 1216 \\
\hline
\end{tabular}

Dos indivíduos com registo de prescrição farmacológica prévia, 66\% foram medicados nos últimos 12 meses, enquanto para os restantes 34\% não foi objectivada na plataforma informática PEM qualquer prescrição de terapêutica antidepressiva no último ano.

Os fármacos antidepressivos mais utilizados pertenciam à classe dos inibidores selectivos da recaptação de serotonina (SSRI), seguindo-se os inibidores da recaptação de serotonina e noradrenalina (SNRI), noradrenérgicos e serotonérgicos específicos (NaSSA) e agonistas da serotonina e inibidores da sua recaptação (SARI) (gráfico 3).

Gráfico 3. Grupos terapêuticos utilizados

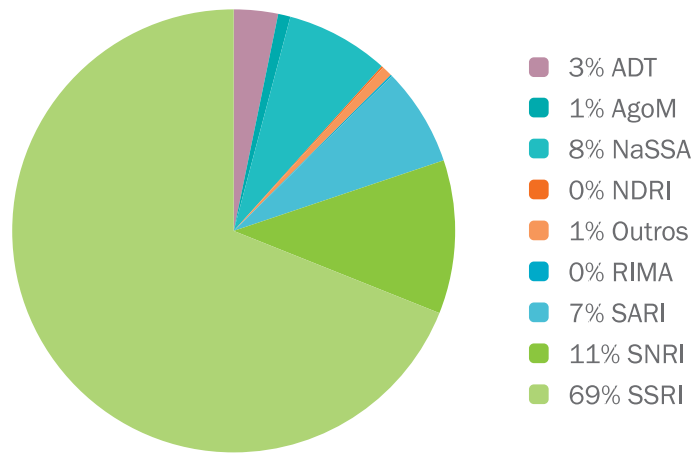

Legenda: ADT - antidepressivos tricíclicos, AgoM - agonista melatoninérgico, NaSSA - noradrenérgicos e serotonérgicos específicos, NDRI - inibidores de recaptação de dopamina e noradrenalina, Outros - tianeptina ou maprotilina, RIMA - inibidor reversível da MAO (monoaminooxidade), SARI - agonistas da serotonina e inibidores da sua recaptação, SNRI - inibidores da recaptação de serotonina e noradrenalina, SSRI - inibidores seletivos da recaptação de serotonina.

\section{Discussão}

Este estudo orientou-se para a avaliação da prevalência de depressão com base nas codificações do registo clínico electrónico (obtida retrospectivamente), pelo que não é possível avaliar se todos os casos correspondem a um diagnóstico correcto desta patologia, pois não se realizou nenhuma avaliação do diagnóstico clínico individual contrastado com outra forma de diagnóstico ou medição de perturbação depressiva. Existe uma possibilidade de sobrediagnóstico, por um conjunto de factores, entre os quais alguma sobrevalorização de parâmetros ou queixas do foro psíquico / somático; Do mesmo modo, neste estudo, não se procurou sistematicamente averiguar se eventuais casos de Depressão Major considerados em remissão parcial poderiam antes ser atribuídos a Perturbação Distímica. ${ }^{3,8}$ Outro dos possíveis factores de sobrediagnóstico é a falha em retirar o código de Depressão dos problemas activos do 
processo clínico, em situações em que terá havido cura clínica e, consequentemente, não existirem já os pressupostos para manutenção dessa classificação.

Não se pode, assim, excluir que devido aos condicionalismos do estudo, a amostra utilizada seja enviesada, o que explicaria uma prevalência de perturbação depressiva na USFR (12\%) mais elevada quando comparada com a prevalência relativa ao ACeSPO, à região norte ou a nível nacional. Alguns autores referem que existe uma tendência para subdiagnosticar a depressão, facto com implicações quanto às decisões terapêuticas. ${ }^{11}$

Comparativamente aos dados nacionais já publicados ${ }^{3,7}$ verifica-se que a prevalência de perturbação depressiva encontrada entre os utentes da USFR é superior em cerca de 50\% à verificada para a população portuguesa, nos estudos referidos, com os reparos manifestados atrás.

A maior prevalência de depressão no género feminino (proporção encontrada de 2,7:1) é um resultado esperado, dado esta perturbação ser mais frequente nas muIheres. A justificação passa por razões de ordem fisiopatológica descritas na literatura (embora não totalmente estabelecidas), podendo também referir-se como factores etiológicos/agravantes de depressão a exigência social imposta à mulher bem como o nível sociocultural. Apontam-se ainda outras causas, que podem enviesar os resultados obtidos, nomeadamente: maior procura de cuidados de saúde pelas mulheres, com ênfase para os CSP: encontra-se descrito no nosso país praticamente o dobro da taxa de utilização de cuidados de saúde pelas mulheres e maior tendência destas a comunicar espontaneamente queixas subjectivas de natureza psíquica. ${ }^{7,9}$

A proporção encontrada entre mulheres e homens na amostra deste estudo foi de 2,7 para 1 . Outros trabalhos referem proporções variáveis entre si, mas concordantes no que se refere à predominância do género feminino. ${ }^{10}$

Em Portugal, as estimativas da prevalência da depressão apontam para diferenças significativas de acordo com a idade, emergindo um padrão de maior expressão quantitativa nos escalões mais jovens, enquanto o escalão de indivíduos acima dos 65 anos apresenta prevalências estimadas significativamente mais baixas que nas restantes faixas etárias, aspecto que segundo os autores poderá estar eventualmente associado um componente de enviesamento de memória (memória selectiva) e comum subvalorização das queixas dos indivíduos idosos por partes dos clínicos. ${ }^{3,12}$
No presente estudo, verificou-se um aumento significativo da prevalência de patologia depressiva apenas após os 30 anos. Entre os 18 e os 30 anos, a prevalência foi de $10,7 \%$ e cerca de $60 \%$ dos utentes com diagnóstico de depressão tinham idades compreendidas entre os 40 e os 69 anos. A este facto poder-se-á associar a carência socioeconómica da população adulta e, sobretudo, idosa da área geográfica que a USFR serve e da sua estreita relação com uma maior debilidade cognitiva e psíquica com consequente susceptibilidade para a depressão. ${ }^{13}$

É também importante salientar que a prevalência da depressão encontrada nos indivíduos com mais de 65 anos foi de aproximadamente $22 \%$, um valor estatisticamente superior quando comparado a outros relatórios nacionais. Este valor poderá relacionar-se com o facto de a população estudada ter maior número de indivíduos naquela faixa etária comparativamente aos estudos citados. ${ }^{3,7}$ Neste âmbito, encontra-se bem documentada a susceptibilidade dos indivíduos idosos para psicopatologia, sendo mesmo aceite como grupo de risco no que se refere a perturbação depressiva. São, neste âmbito, apontadas como possíveis causas a deterioração física e cognitiva relacionadas com o factor idade, bem como a menor ocupação diária e o isolamento social. ${ }^{9}$

Quase um terço dos indivíduos em estudo apresentava diagnóstico activo há mais de 5 anos, muitos desde o início dos registos informáticos. A este facto os investigadores atribuem hipóteses causais explicando a duração prolongada da patologia tais como: parte daqueles diagnósticos ainda se manter, alguns casos corresponderem a recaídas, tratar-se de outras patologias depressivas descritas igualmente em vários trabalhos (integradas no mesmo código da ICPC-2) como distimia ou perturbação bipolar. Poderão ainda tratar-se de episódios já terminados, mas que o médico não transpôs para inactivos, ou meros erros de codificação.

Cerca de $12 \%$ dos utentes estudados não tinha consulta há mais de um ano, colocando-se em causa, nestes casos, o acompanhamento adequado dos indivíduos com depressão, muito embora se possam considerar vieses que justifiquem estes dados: possibilidade de diagnóstico incorrecto, erros de codificação, diagnósticos já não activos mas que não foram transpostos para inactivos, utentes com depressão que não compareceram a consulta quando convocados ou ausência de convocatória - parâmetros não avaliados pelos investigadores. 
Constitui ainda uma debilidade deste estudo o facto de em caso de consulta presencial há menos de 12 meses não ter sido verificado se o problema "depressão" foi abordado/codificado na mesma consulta, o que permitiria detectar problemas como a má qualidade de registos, não codificação ou a não abordagem do assunto durante o contacto médico-doente, não significando assim necessariamente o acompanhamento adequado da patologia em causa.

Aproximadamente $18 \%$ dos indivíduos com diagnóstico activo não estavam medicados com terapêutica antidepressiva. Este resultado poderá ser explicado pelo facto de os utentes deixarem de cumprir o tratamento farmacológico por vontade própria ou pela não abordagem do problema por parte dos clínicos nos diferentes contactos com os utentes (poder-se-á apontar esquecimento, má qualidade dos registos ou o tempo disponível para a consulta) condicionando, assim, a ausência de prescrição daquelas terapêuticas.

Para cerca de um terço dos indivíduos com prescrição prévia não foi observada qualquer prescrição de terapêutica antidepressiva nos 12 meses prévios à recoIha dos dados. Confrontando os resultados relativos ao "tempo decorrido desde a última consulta" a estes últimos, poder-se-á inferir que alguns dos utentes que não têm prescrição há mais de um ano poderão corresponder a alguns utentes que não tiveram consulta no mesmo período.

Poder-se-á, igualmente, retirar importantes conclusões quando observados os dados relativos ao local de prescrição dos psicofármacos. Como é conhecido, os CSP são a porta de acesso à saúde da grande maioria dos utentes do Serviço Nacional de Saúde (SNS), facto semelhante na maioria dos países. É, portanto, a nível primário que a larga maioria dos diagnósticos de depressão são hoje realizados e, neste contexto, é onde se efectua o seguimento médico dos utentes que sofrem desta patologia, salvo as devidas excepções merecedoras de referenciação para os Cuidados de Saúde Secundários. No presente estudo, cerca de $78 \%$ do total de prescrições medicamentosas foram realizadas na USFR, enquanto as restantes se dividiam entre o Hospital de referência em cuidados de Psiquiatria e de Saúde Mental da área geográfica da unidade de saúde e outras entidades privadas.

Realça-se, assim, o papel do Médico de Família que, pela sua privilegiada posição face ao utente e estreito contacto com os seus factores pessoais e ambientais, pode ser relevante na detecção adequada e atempada de psicopatologia, nomeadamente de perturbação depressiva.
Os fármacos antidepressivos mais utilizados pertenciam à classe dos SSRI. Segundo as mais recentes normas de orientação clínica, qualquer fármaco antidepressivo poderá ser utilizado no tratamento da depressão desde que a sua escolha tenha como base a sua tolerabilidade, segurança, menor custo, bem como a história individual do doente, as suas preferências e os antecedentes de resposta a tratamentos realizados anteriormente, não existindo diferenças significativas quanto à eficácia.

Dada a menor amplitude e gravidade de efeitos secundários, assim como a boa tolerabilidade e perfil de segurança cardiovascular mais favorável atribuídos aos SSRI, estes justificam assim a sua escolha em maior escala pela generalidade dos clínicos nos últimos anos, facto também observado neste estudo. ${ }^{12}$

Em conclusão, a falta de estudos epidemiológicos de base populacional sobre a prevalência de perturbações mentais está bem patente na escassa literatura existente, particularmente em Portugal. Assim, as consequências deste estudo poderão ser positivas se os resultados forem adequadamente enquadrados e entendidos.

Este estudo, cuja validade se baseia no tratamento de dados com satisfatória cobertura populacional, permitiu conhecer a taxa de prevalência de depressão entre os utentes da USFR, mais elevada que o esperado. Os autores encontraram ainda diferenças entre géneros, com as mulheres a apresentarem níveis mais elevados de depressão. Foi possível estabelecer populações de risco quanto à idade, com a existência de dois picos, entre a quinta e sexta década e nos indivíduos com mais de 65 anos.

Os resultados não dão todas as respostas e produzem novas questões, devendo ser vistos à luz dos seus limites. É de fundamental importância delinear estratégias futuras junto dos profissionais de saúde, de forma a clarificar sintomas e critérios de diagnóstico, com o objectivo de melhorar os cuidados à população e obter ganhos em saúde.

\section{Agradecimentos}

Os autores agradecem aos colegas Dra. Carla Silva, Dra. Iryna Mykolyshyn, Dra. Lígia Sousa e Dr. Pedro Sousa a colaboração no processo de colheita de dados. 


\section{Referências Bibliográficas}

1. World Health Organization. Mental health: facing the challenges, building solutions: report from the WHO European Ministerial Conference, 2005. Acesso a 23 Abril, 2016.

2. Gusmão, Xavier, Heitor, Bento, \& Almeida. Depressive disorder burden: global epidemiological issues and information needs in Portugal. Acta Med Port. 2005 MarApr;18(2):129-46

3. Almeida JMC, Xavier M. Estudo Epidemiológico Nacional de Saúde Mental - $1 .^{\circ}$ Relatório. 2013. Faculdade de Ciências Médicas, Universidade Nova de Lisboa.

4. Apóstolo J, Alves L, Figueiredo MH, et al. Depression, anxiety and stress in primary health care users. Revista Latino-Americana de Enfermagem; 2011; 19(2), 348-353.

5. Bebbington, P. E. The origins of sex differences in depressive disorder: bridging the gap. International Review of Psychiatry, 1996. 8, 295 -332.

6. Bebbington, P. E. Sex and depression. Psychological Medicine, 1998. 28, 1-8.

7. Direção-Geral da Saúde (Março.2016). Saúde Mental em Números 2015. Lisboa.

8. Gonçalves B, Fagulha T, Ferreira A. A depressão nas mulheres de meia idade: estudo sobre as utentes dos cuidados de saúde primários. Psicologia; 2005; 19(1-2), 39-56.

9. Resina T, Pinhão A, Neto L, et al. Depressão e ansiedade na consulta de clínica geral/ médico de família. Acta Méd. Port. 1989; 2(1):7-10.

10. World Health Organization. The world health report 2001 - Mental Health: new understanding, new hope. Geneva: World Health Organization; 2001.

11. Gonçalves B, Fagulha T, Prevalência e Diagnóstico da Depressão em Medicina Geral e Familiar, Rev. Port. Clín. Geral 2004; 20:13-27.

12. Direção-Geral de Saúde. Circular Normativa n . 034/2012 - Terapêutica Farmacológica da Depressão Major e da sua Recorrência no Adulto de 30/12/2012, disponível em https://www.dgs.pt/directrizes-da-dgs/normas-ecirculares-normativas/norma-n-0342012-de-30122012. aspx. Acesso a 1 Março, 2016.

13. InstitutoNacionaldeEstatísticaCensos2011. Disponívelem https://www.ine.pt/xportal/xmain?xpid=INE\&xpgid=ine_ bdc_tree\&contexto=bd\&selTab=tab2 (Acesso em 29 de Setembro de 2017)

\section{Conflito de Interesses}

Os autores declaram não ter conflitos de interesses. 